\title{
Article \\ Effects of a 6-Minute Fast-Walking Protocol on Changes in Muscle Activity in Individuals with Flatfoot
}

\author{
Chu-Yuan Chiang, Kuang-Wei Lin (D), Hui-Min Lee, Shun-Hwa Wei and Li-Wei Chou *(D)
}

Citation: Chiang, C.-Y.; Lin, K.-W.; Lee, H.-M.; Wei, S.-H.; Chou, L.-W. Effects of a 6-Minute Fast-Walking Protocol on Changes in Muscle Activity in Individuals with Flatfoot. Appl. Sci. 2022, 12, 2207. https:// doi.org/10.3390/app12042207

Academic Editors: Alessandro de Sire and Ozden Ozyemisci Taskiran

Received: 23 December 2021

Accepted: 18 February 2022

Published: 20 February 2022

Publisher's Note: MDPI stays neutral with regard to jurisdictional claims in published maps and institutional affiliations.

Copyright: (C) 2022 by the authors. Licensee MDPI, Basel, Switzerland. This article is an open access article distributed under the terms and conditions of the Creative Commons Attribution (CC BY) license (https:// creativecommons.org/licenses/by/ $4.0 /)$.
Department of Physical Therapy and Assistive Technology, National Yang Ming Chiao Tung University, Taipei 112, Taiwan; 8chiang8@gmail.com (C.-Y.C.); wade1112@gm.ym.edu.tw (K.-W.L.); noralee0724@gmail.com (H.-M.L.); shunhwa@nycu.edu.tw (S.-H.W.)

* Correspondence: lwchou@nycu.edu.tw; Tel.: +886-2-2826-7092

\begin{abstract}
Flatfoot causes abnormal biomechanics in the lower extremity, resulting in discomfort and excessive burden on lower extremity muscles during functional tasks, and it potentially leads to associated syndromes in the lower extremity. The aim of this study was to investigate how a demanding, repetitive task affects the muscle strength, activities, and fatigue of the lower extremities during function tasks. Nineteen individuals with flexible flatfoot (10M9F, age: $24.74 \pm 2.68$ years) and fifteen non-flatfoot participants (6M9F, age: $24.47 \pm 3.74)$ took part in this study. All participants performed maximal voluntary isometric contraction and functional tasks, including walking and single-leg standing tests before and immediately after a 6-min fast-walking protocol. A surface electromyography system was used to collect muscle activation data. Our results showed that, after 6 min of fast walking, peroneus longus activity increased only in the non-flatfoot group, and gastrocnemius activity increased in the flexible flatfoot group. In the flexible flatfoot group, greater recruitment in abductor halluces and greater fatigue in the tibialis anterior was observed. Individuals with flexible flatfoot showed altered muscle activation pattern after 6-min fast walking. These findings can provide an evidence-based explanation of associated syndromes in flatfoot populations and lead to potential intervention strategies in the future.
\end{abstract}

Keywords: flexible flatfoot; biomechanics; muscle activity; six-minute fast walk

\section{Introduction}

The foot is constituted of 26 bones connected by multiple joints, ligaments, and tendons. Foot problems may cause inflammation and pain, which do have a negative impact on quality of life [1]. Flatfoot, also termed pes planus, is a postural deformity in which the arches of the foot collapse. Common symptoms include attenuation, insufficiency, or rupture of the supporting structures of the medial longitudinal arch (MLA) [2]. Statistics show that the prevalence of flexible flatfoot was $13.6 \%$ in adulthood [3], and surgery might be required for severe cases [4].

The disorder gives rise to abnormal biomechanics, including hindfoot valgus, forefoot abduction, abnormal internal rotation of the talus and calcaneus, and increased internal rotation of the tibia $[2,5,6]$. Therefore, individuals with flatfoot require more muscle action than non-flatfooted people to support and propel the weight of their bodies due to instability in foot structure [7]. During walking, changes in muscle activation occur in different phases and different muscles; this includes a higher percentage of peak surface electromyography (sEMG) amplitude by the tibialis anterior in the contact phase and the tibialis posterior during propulsion, but a lower percentage on the peroneus longus throughout the stance phase [8]. Individuals with flatfoot also show higher tibialis anterior activity and lower activity in the peronei, soleus, and gastrocnemius in early stance relative to the mean stance phase [9].

Less than $1 \%$ of individuals with flexible flatfoot are symptomatic [10]. A flattened arch results in overpronation, leading to changes in foot structure. Therefore, problems may 
extend to the proximal joint. Some studies have revealed that a higher prevalence of anterior knee pain, intermittent lower back pain, medial tibial stress syndrome, and knee cartilage damage is associated with having flat feet [11-13]. The abnormal biomechanics of flatfoot causes ankle instability, which can, in turn, lead to knee, hip, or even trunk problems.

A previous electromyographic study revealed that the activity of the abductor hallucis decreased corresponded with an increase in navicular drop, signifying that this muscle plays an important role in supporting the MLA [14]. Another study involved specifically causing fatigue in this muscle; that study revealed increases in navicular drop that resulted in foot pronation [15]. Muscle fatigue increasing the amount of biomechanical changes of flatfoot might cause associated syndromes and other injuries.

Most previous studies have compared biomechanical differences between non-flatfoot and flatfoot $[5,9,16]$, but few studies have focused on the contribution of muscle overuse to biomechanical changes related to flatfoot. Although some fatigue protocols have been used in studies as interventions on both non-flatfooted and flatfooted individuals, those protocols do not typically represent daily activities.

Pathological mechanisms of flatfoot-associated syndromes remain unclear, as do the alterations of lower-extremity biomechanics for performing functional tasks. In order to understand the mechanisms of fatigue on lower-limb muscles, the purpose of our study was to assess changes in tibialis anterior, gastrocnemius, peroneus longus, and abductor hallucis muscle strength, activities, and the phenomenon of fatigue during functional tasks before and immediately after a 6-min fast-walk protocol in non-flatfoot individuals versus those with flatfoot. Our hypotheses were that the protocol would decrease muscle strength, increase muscle activation, and decrease sEMG median frequency after a 6-min fast-walk protocol.

\section{Materials and Methods}

This case-control study was conducted to evaluate the changes of lower-limb muscles after a 6-min fast-walk protocol. Data were collected during the period from April 2017 to November 2017 in a motion analysis laboratory at National Yang Ming Chiao Tung University, Taipei, Taiwan. Figure 1 shows the study flowchart. Fifteen non-flatfoot participants and nineteen with flexible flatfoot, all between the ages of 20 and 65 years, were recruited. Individuals with flexible flatfoot were distinguished from non-flatfoot individuals, using a navicular drop test, which is commonly used in clinical settings, as it can reveal the ability of the MLA to act as a supportive structure. The definition of flatfoot in this study is greater than $1 \mathrm{~cm}$ height difference of the navicular tuberosity between subtalar neutral and relaxed stance positions [17]. The inclusion criteria for the flatfoot group were established as flatfoot by the navicular drop test. We excluded subjects who self-reported a history of lower-extremity injuries within the previous 6 months, any lower-extremity surgery, and occurrence of neurological disease prior to participation. Subjects with skin allergy related to alcohol swabs and electrode pads were also excluded. This study was approved by the ethics committee of National Yang-Ming University (YM105056E) and conducted according to the Declaration of Helsinki. All participants' written informed consent was obtained before the experiments. We recorded each participant's height, weight, age, sex, and dominant foot; only the non-dominant foot was tested in the experiment. International Physical Activity Questionnaires (IPAQs) were used to assess each participant's daily activity level.

The 6-min fast walking on the treadmill was the fatigue protocol in this study. Participants were instructed to walk on the treadmill with self-selected speed for 3 min to familiarize and warm up with the task. To estimate the preferred walk-run transition speed, the treadmill speed was increased gradually. The preferred walk-run transition speed was reduced by $0.1 \mathrm{~km} / \mathrm{h}$ as the test speed so that participants could maintain walking for 6 min during the protocol. 


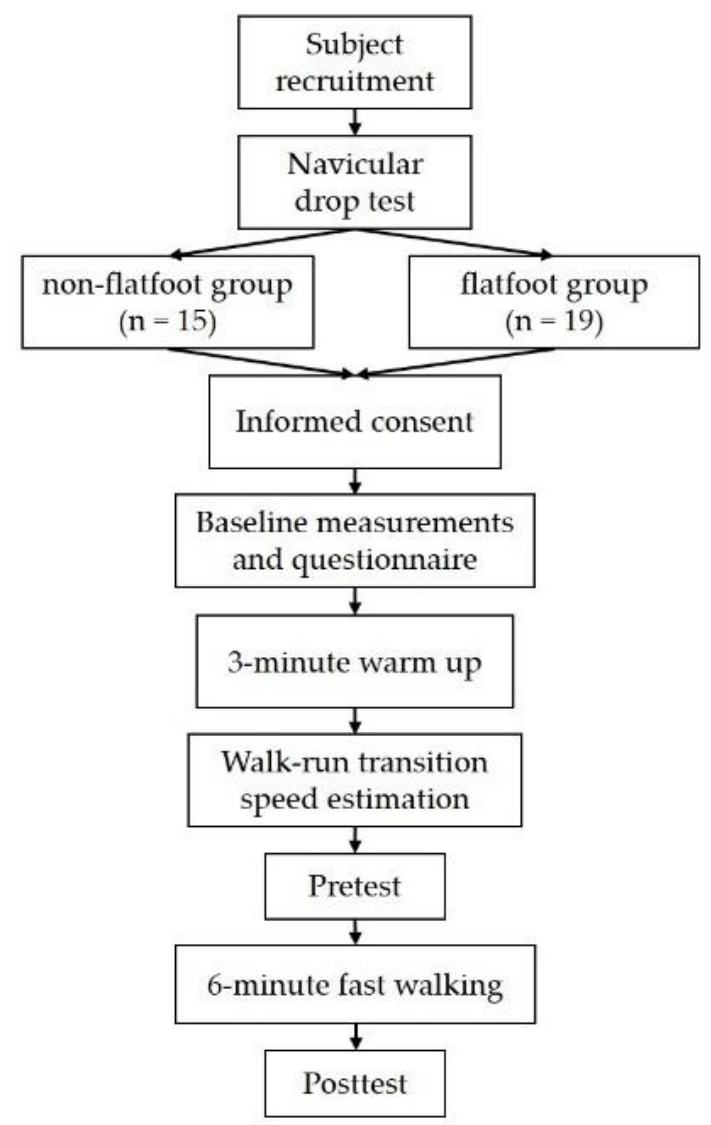

Figure 1. Study flowchart.

A motion capture system (Vicon Motion Systems Ltd., Oxford, UK) with eight infrared cameras operating at $100 \mathrm{~Hz}$ was used to capture and determine gait cycle. The Plug-In-Gait model was used; however, the kinematic data would process for further study. A force plate (AMTI, Advanced Mechanical Technology Inc., Watertown, MA, USA) operating at $1000 \mathrm{~Hz}$ was used to synchronically record ground reaction forces during tasks. The heel strike of level walking was determined at the first frame at which the vertical ground reaction force was larger than 20 N. A sEMG system (MP150, BIOPAC Systems Inc., Goleta, CA, USA) operating at $1000 \mathrm{~Hz}$ was used to collect muscle activation data synchronically with the motion capture system. Before and after the $6 \mathrm{~min}$ of fast walking, the maximal voluntary isometric contraction (MVIC) in the tibialis anterior, gastrocnemius, abductor halluces, and peroneus longus would be measured. Two functional tasks, namely level walking and single-leg standing tests, were executed in the same order.

Maximal muscle strength assessment was performed by using manual muscle testing with a handheld dynamometer. To test the tibialis anterior, the participant was supine on the treatment table, and the dominant leg with knee flexion and hip external rotation placing underneath the popliteal fossa of the test foot and test foot performed ankle dorsiflexion (Figure 2a). To test the gastrocnemius, the participant was asked to lie prone with knee flexion $90^{\circ}$ and performed ankle plantarflexion (Figure 2b). Peroneus longus was tested in a side-lying position and the dominant leg with hip and knee in flexion position placing under the test leg. Then the participant performed foot eversion in this position (Figure 2c). For the abductor hallucis, however, the maximal muscle strength cannot be assessed by the handheld dynamometer. Therefore, each participant was asked to sit on a chair with the hip, knee, and ankle at approximately $90^{\circ}$ and was asked to scratch the paper under the foot and not tighten any other muscles in their legs or trunk during the abductor hallucis MVIC test. In addition, all muscle activations were measured during MVIC tests. 

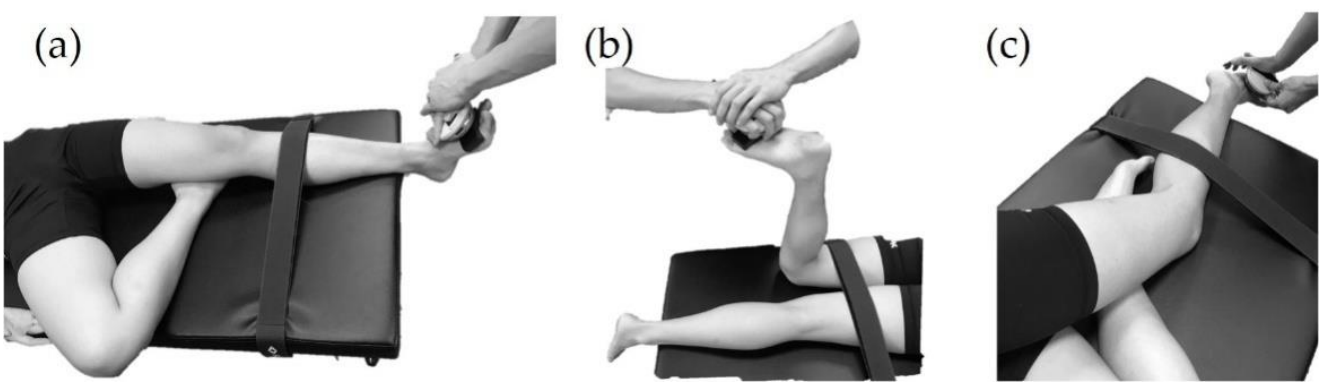

Figure 2. Body position during maximum voluntary isometric contraction (MVIC) test. (a) MVIC testing of the tibialis anterior. (b) MVIC testing of the gastrocnemius. (c) MVIC testing of the peroneus longus.

All the subjects performed the following 2 functional tasks before and after the 6-min fast walking. The first functional task was level walking. The participants performed five trials of $10 \mathrm{~m}$ barefoot walking at self-selected speed. The second functional task was single-leg standing for $20 \mathrm{~s}$. Participants were asked to perform single-leg standing with the non-dominant leg and cross their arms over the front of their chest. The dominant leg was to perform 90 degrees of knee flexion, and sEMG was recorded during these tasks; the MVIC data were used to normalize sEMG amplitudes.

All data were processed by using a custom-written software program (MATLAB 2018a; MathWorks, Inc., Natick, MA, USA). For the MVIC tasks, three-second sEMG data were selected for further analysis. The median frequency was determined by using fast Fourier transformation, with a 1024 data-point window moving over the whole selected signal area, and a 512 data point shift. A decreased median frequency was an indication of muscle fatigue [18]. For the functional tasks, raw sEMG signals were filtered with a bandwidth filter between 10 and $450 \mathrm{~Hz}$ and smoothed by using a moving root mean square filter (20 ms time constant). The peak amplitudes of tasks were normalized by MVIC sEMG data.

Participants' baseline data were represented as mean \pm standard deviation (SD). The continuous variables (including age, height, and weight) were compared with independent t-tests. Multivariate analysis of variance (MANOVA) was performed on sEMG parameters to determine the main effects between groups (non-flatfoot and flatfoot) and within groups (pretest vs. posttest). Significant differences revealed by the MANOVA were examined further by using one-way repeated measures ANOVA and Bonferroni's post hoc analysis. The level of significance was set at $\alpha=0.05$ for all analyses. All statistical analyses were performed with SPSS 20.0 software (SPSS Inc., Chicago, IL, USA).

\section{Results}

The demographic data (Table 1) showed significant differences in body mass index (BMI) and navicular drop between non-flatfoot and flatfoot groups. BMI in flatfoot group was higher than in the non-flatfoot group. These results matched previous epidemiological study observations.

After 6 min of fast walking, with the exception of the peroneus longus in the flatfoot group, MVIC of all the other tested muscles showed significant decrease, suggesting our 6-min fast-walking protocol required a significant amount of workload and caused muscle fatigue. Among all the tested muscles, MVIC of the tibialis anterior decreased the most, it dropped 32\% and 35\% for non-flatfoot and flatfoot groups, respectively (Figure 3). 
Table 1. Baseline demographic data.

\begin{tabular}{lccc}
\hline \multicolumn{1}{c}{ Variable } & $\begin{array}{c}\text { Non-Flatfoot } \\
\text { (Mean } \pm \text { SD) }\end{array}$ & $\begin{array}{c}\text { Flatfoot } \\
\text { (Mean } \pm \text { SD) }\end{array}$ & $p$-Value \\
\hline Demographic measurements & & & \\
Age (year) & $24.47 \pm 3.74$ & $24.74 \pm 2.68$ & 0.81 \\
Height $(\mathrm{m})$ & $1.68 \pm 0.07$ & $1.69 \pm 0.08$ & 0.61 \\
Weight $(\mathrm{kg})$ & $58.87 \pm 9.78$ & $66.5 \pm 12.89$ & 0.07 \\
Body mass index $\left(\mathrm{kg} / \mathrm{m}^{2}\right)$ & $20.71 \pm 2.53$ & $22.91 \pm 2.56$ & $0.02^{*}$ \\
Navicular drop $(\mathrm{cm})$ & $0.70 \pm 0.15$ & $1.22 \pm 0.19$ & $<0.001^{*}$ \\
IPAQ & 1 & & \\
Low activity & 7 & 6 & \\
Enough & 7 & 12 & \\
High activity & $3307.13 \pm 2434.97$ & $4694.42 \pm 3735.24$ & 0.22 \\
Total (METs) & & & \\
\hline
\end{tabular}

* Significant differences between groups. IPAQ, International Physical Activity Questionnaires; METs, metabolic equivalents.
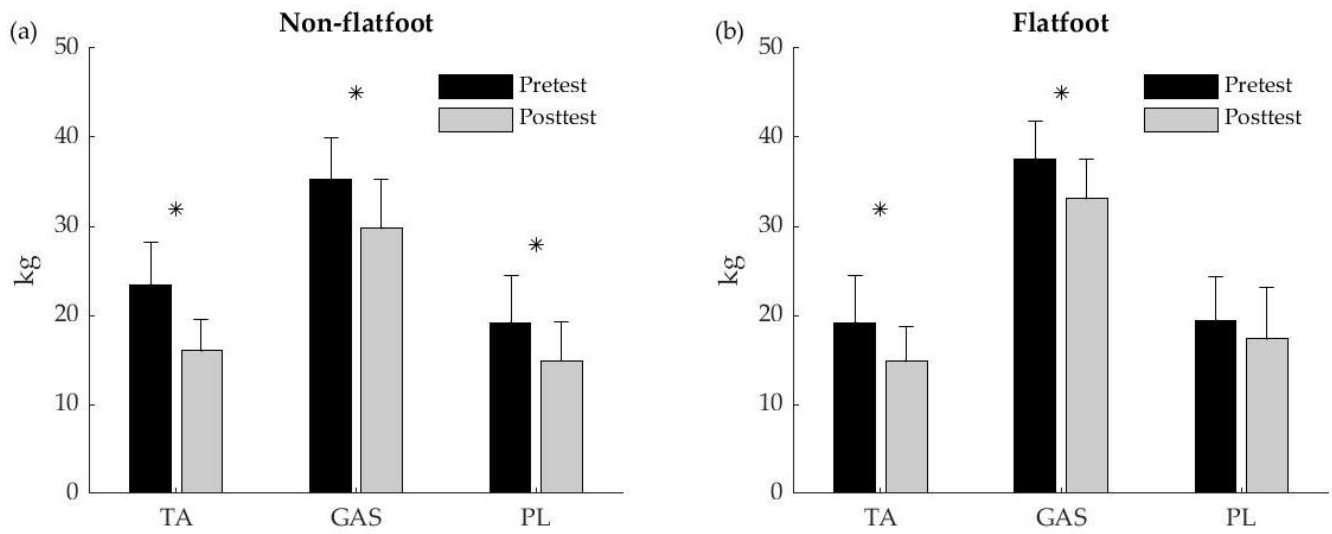

Figure 3. Maximal muscle strength of (a) non-flatfoot and (b) flatfoot groups. * Significant differences between pretest and posttest. TA, tibialis anterior; GAS, gastrocnemius; PL, peroneus longus.

The sEMG amplitude of both function tasks had significant differences within groups (pretest and posttest) but no significant differences between groups (non-flatfoot and flatfoot). Figure 4 showed the percentage of changes in sEMG amplitude between pretest and posttest during walking and single-leg standing tasks. The changes of muscle-activation level of the peroneus longus were significantly higher in the non-flatfoot group with an increase of $36 \%$ compared to the pretest (Figure $4 a$ ). For the flatfoot group, the changes of muscles-activation level of the gastrocnemius were higher with an increase of $62 \%$. In the single-leg standing task, both non-flatfoot and flatfoot participants showed greater gastrocnemius muscle activation ( $44 \%$ and $48 \%$ for non-flatfoot and flatfoot) compared with pretest (Figure 4b). Interestingly, after 6 min of fast walking, the non-flatfoot group showed a tendency of greater abductor hallucis activation ( $43 \%$ increase), whereas the flatfoot group showed a tendency to decrease in abductor hallucis activation ( $21 \%$ decrease) in single-leg standing.

The median frequency of the tibialis anterior in flatfoot group was significantly decreased (from 116 to $106 \mathrm{~Hz}$ ) after 6 min of fast walking ( $p<0.001$ ), further confirming that the tibialis anterior was likely most fatigued (Figure 5). Interestingly, the median frequency of the abductor hallucis in the flatfoot group increased (from 189 to $215 \mathrm{~Hz}$ ) after 6 min of fast walking $(p<0.001)$. 

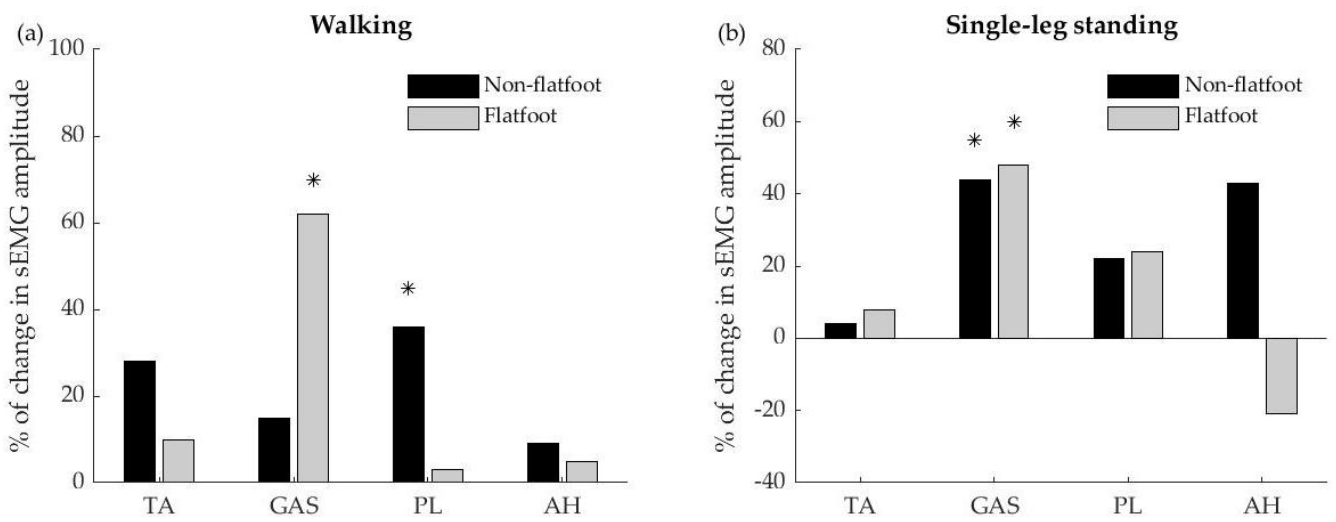

Figure 4. Changes of muscle-activation level during (a) walking and (b) single-leg standing for non-flatfoot and flatfoot groups. * Significant differences between pretest and posttest. TA, tibialis anterior; GAS, gastrocnemius; PL, peroneus longus; $\mathrm{AH}$, abductor hallucis.
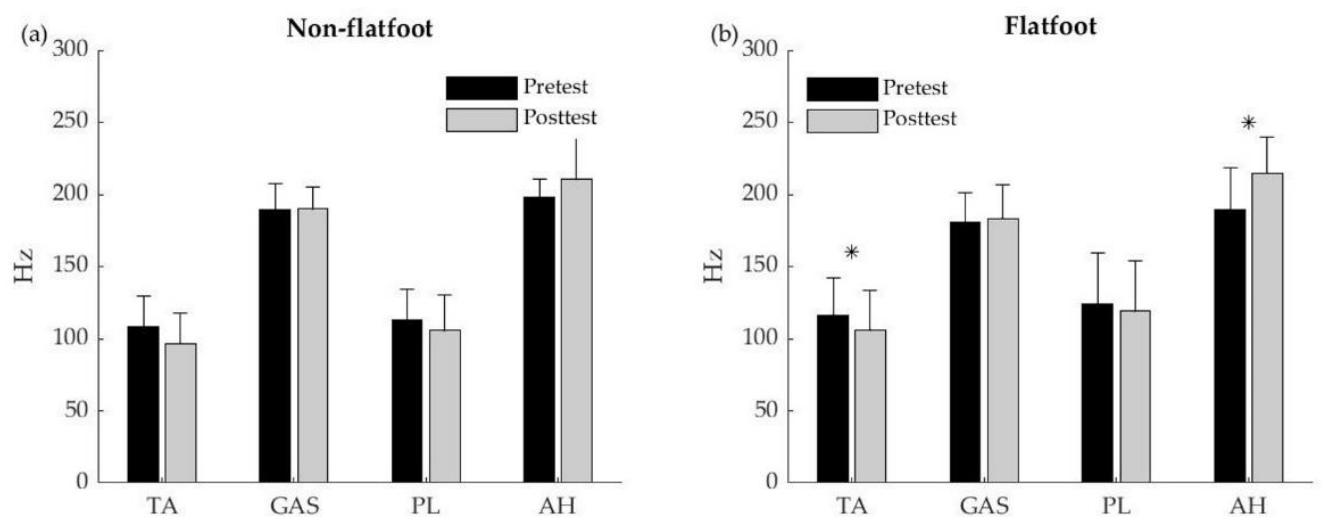

Figure 5. Median frequency for (a) non-flatfoot and (b) flatfoot groups. * Significant differences between pretest and posttest. TA, tibialis anterior; GAS, gastrocnemius; PL, peroneus longus; AH, abductor hallucis.

\section{Discussion}

This study aimed to assess the changes in lower-limb muscle activities during functional tasks before and immediately after a 6-min fast-walk protocol in individuals with flatfoot and non-flatfoot. The key finding of this study is that, in the flatfoot group, sEMG median frequency decreased for the tibialis anterior and increased for the abductor hallucis after a 6-min fast-walk task, suggesting fatigue in the tibialis anterior and recruitment in the abductor hallucis after the protocol. Both groups showed increased gastrocnemius activity during the single-leg standing test. In the walking test, peroneus longus activation increased in the non-flatfoot group, whereas gastrocnemius activity increased in the flatfoot group.

Previous studies measured the biomechanical parameters in individuals of flatfoot under static or dynamic conditions without fatigue protocol as an intervention $[7,9,19]$. To our knowledge, this is the first study to use such a demanding functional protocol in an attempt to emulate the high-loading conditions of daily life; however, some studies assessed the effect of foot intrinsic muscle fatigue by different protocols $[15,20]$. In this study, we used a 6-min fast-walk protocol to investigate changes in muscle activation of individuals with flatfoot and compared them with those of non-flatfoot adults. Following the protocol, both groups showed considerably decreased muscle strength and significant decreases in MVIC, demonstrating that this high-load protocol was sufficiently challenging for both groups. By comparing muscle activation during functional tasks between individuals with and without flatfoot, this study demonstrated that demanding repetitive motor tasks could 
increase muscle recruitment and alter muscle-activation level to compensate and provide support to the MLA in individuals with flatfoot.

We observed that, after the fast-walk protocol, the tibialis anterior is fatigued (decreased EMG median frequency), and its strength is decreased (MVIC). As an invertor, the tibialis anterior contracts to prevent eversion and provide joint stability during walking. Loss of arch support and greater extrinsic muscle activation for postural control occurred in flatfoot individuals. Hence, individuals with flatfoot required greater gastrocnemius activation for generating propulsion force during walking. As part of the triceps surae complex, gastrocnemius with higher activation during walking could result in powerful pronation force during the heel-rise phase, which increases tension in the Achilles tendon by $30 \%$ and causes excessive tension in the triceps surae $[19,21]$, as well as causing early heel rise during walking [7]. In addition, we also found that the flatfoot group had increased peroneus longus activation after $6 \mathrm{~min}$ of fast walking. Altered triceps surae activation [22], early heel rise during walking [23], and unbalanced ankle inversion/eversion strength [24] have been associated with medial tibial stress syndrome. Our results indicated that a demanding repetitive walking task could cause altered lower-extremity muscle activation in individuals with flatfoot, and this could lead to the development of associated syndromes, such as medial tibial stress syndrome.

Although the AH muscle did not show significant changes in EMG amplitude (activation level) during walking or single leg stance after the 6-min fast-walking protocol, the AH muscle did show an increase in EMG median frequency. It is generally believed that an increase in EMG median frequency is caused by increasing average conduction velocity during motor unit recruitment $[25,26]$. Activation of the AH muscle is crucial for controlling the deformation of the longitudinal arch [27]. Demanding repetitive motor tasks, such as fast walking, might induce motor unit recruitment of the AH muscle in individuals with flatfoot. Physical therapy, motor training, and custom foot orthoses are common treatment options for patients with flatfoot [28]. Future studies could further investigate whether and how motor tasks, such as fast walking, could help develop and become an effective training protocol for individuals with flatfoot.

There were limitations to this study. First, we only measured activation in four superficial muscles. Deeper muscles, such as the tibialis posterior, may also exhibit an altered activation pattern after 6 min of fast walking. A future study with invasive recording technique or musculoskeletal modeling is needed to establish a more complete view of how lower-extremity muscles change their activation pattern to compensate for repetitive demanding functional tasks in individuals with functional flatfoot, and how these changes link to associated syndrome in the lower extremity. Second, the participants in the flatfoot group had mainly mild-to-moderate flatfoot, which is asymptomatic and without any severe deformations. The level of severity might be a factor influencing outcome measures that limits the results of the current study to be applied to individuals with more severe flatfoot. Future research concerning mechanisms of associated syndromes could study activation patterns of both superficial and deep muscles, as well as kinetic/kinematic changes of lower extremity under overground walking condition, which may help better elucidate these mechanisms and lead to more effective preventive or therapeutic measures.

\section{Conclusions}

The muscle activation differed between flatfoot and non-flatfoot adults after the 6-min fast-walk protocol. Demanding and repetitive motor tasks, such as fast walking, increased gastrocnemius activation, thus suggesting a greater degree of extrinsic muscle recruitment to compensate and support ankle and foot stability in individuals with flatfoot.

Author Contributions: Conceptualization, C.-Y.C. and L.-W.C.; data curation, K.-W.L.; funding acquisition, L.-W.C.; methodology, C.-Y.C. and H.-M.L.; resources, S.-H.W.; supervision, L.-W.C.; validation, S.-H.W.; writing-original draft, C.-Y.C. and K.-W.L.; writing—review and editing, H.-M.L. and L.-W.C. All authors have read and agreed to the published version of the manuscript. 
Funding: This research was funded by Ministry of Science and Technology, Taiwan, grant number MOST 110-2221-E-A49A-501-MY3.

Institutional Review Board Statement: This study was approved by the ethics committee of National Yang-Ming University (YM105056E) and conducted according to the Declaration of Helsinki.

Informed Consent Statement: Written informed consent was obtained from all subjects involved in the study.

Data Availability Statement: The data presented in this study are available upon request from the corresponding author.

Conflicts of Interest: The authors declare no conflict of interest.

\section{References}

1. López-López, D.; Pérez-Ríos, M.; Ruano-Ravina, A.; Losa-Iglesias, M.E.; Becerro-de-Bengoa-Vallejo, R.; Romero-Morales, C.; Calvo-Lobo, C.; Navarro-Flores, E. Impact of quality of life related to foot problems: A case-control study. Sci. Rep. 2021, 11, 14515. [CrossRef] [PubMed]

2. Lever, C.J.; Hennessy, M.S. Adult flat foot deformity. Orthop. Trauma 2016, 30, 41-50. [CrossRef]

3. Aenumulapalli, A.; Kulkarni, M.M.; Gandotra, A.R. Prevalence of flexible flat foot in adults: A cross-sectional study. J. Clin. Diagn. Res. 2017, 11, 17-20. [CrossRef] [PubMed]

4. Catani, O.; Cautiero, G.; Sergio, F.; Cattolico, A.; Calafiore, D.; de Sire, A.; Zanchini, F. Medial displacement calcaneal osteotomy for unilateral adult acquired flatfoot: Effects of minimally invasive surgery on pain, alignment, functioning, and quality of life. J. Foot Ankle Surg. 2021, 60, 358-361. [CrossRef] [PubMed]

5. Levinger, P.; Murley, G.S.; Barton, C.J.; Cotchett, M.P.; McSweeney, S.R.; Menz, H.B. A comparison of foot kinematics in people with normal-and flat-arched feet using the Oxford Foot Model. Gait Posture 2010, 32, 519-523. [CrossRef]

6. Lundberg, A.; Svensson, O.K.; Bylund, C.; Selvik, G. Kinematics of the ankle/foot complex-Part 3: Influence of leg rotation. Foot Ankle 1989, 9, 304-309. [CrossRef]

7. Franco, A.H. Pes cavus and pes planus: Analyses and treatment. Phys. Ther. 1987, 67, 688-694. [CrossRef]

8. Murley, G.S.; Menz, H.B.; Landorf, K.B. Foot posture influences the electromyographic activity of selected lower limb muscles during gait. J. Foot Ankle Res. 2009, 2, 35. [CrossRef]

9. Hunt, A.E.; Smith, R.M. Mechanics and control of the flat versus normal foot during the stance phase of walking. Clin. Biomech. 2004, 19, 391-397. [CrossRef]

10. Yeagerman, S.E.; Cross, M.B.; Positano, R.; Doyle, S.M. Evaluation and treatment of symptomatic pes planus. Curr. Opin. Pediatr. 2011, 23, 60-67. [CrossRef]

11. Kosashvili, Y.; Fridman, T.; Backstein, D.; Safir, O.; Ziv, Y.B. The correlation between pes planus and anterior knee or intermittent low back pain. Foot Ankle Int. 2008, 29, 910-913. [CrossRef] [PubMed]

12. Gross, K.D.; Felson, D.T.; Niu, J.; Hunter, D.J.; Guermazi, A.; Roemer, F.W.; Dufour, A.B.; Gensure, R.H.; Hannan, M.T. Association of flat feet with knee pain and cartilage damage in older adults. Arthritis Care Res. 2011, 63, 937-944. [CrossRef] [PubMed]

13. Moen, M.; Bongers, T.; Bakker, E.; Zimmermann, W.; Weir, A.; Tol, J.; Backx, F. Risk factors and prognostic indicators for medial tibial stress syndrome. Scand. J. Med. Sci. Sports 2012, 22, 34-39. [CrossRef] [PubMed]

14. Fiolkowski, P.; Brunt, D.; Bishop, M.; Woo, R.; Horodyski, M. Intrinsic pedal musculature support of the medial longitudinal arch: An electromyography study. J. Foot Ankle Surg. 2003, 42, 327-333. [CrossRef] [PubMed]

15. Headlee, D.L.; Leonard, J.L.; Hart, J.M.; Ingersoll, C.D.; Hertel, J. Fatigue of the plantar intrinsic foot muscles increases navicular drop. J. Electromyogr. Kinesiol. 2008, 18, 420-425. [CrossRef]

16. Kim, J.-A.; Lim, O.-B.; Yi, C.-H. Difference in static and dynamic stability between flexible flatfeet and neutral feet. Gait Posture 2015, 41, 546-550. [CrossRef]

17. Sell, K.E.; Verity, T.M.; Worrell, T.W.; Pease, B.J.; Wigglesworth, J. Two measurement techniques for assessing subtalar joint position: A reliability study. J. Orthop. Sports Phys. Ther. 1994, 19, 162-167. [CrossRef]

18. Horita, T.; Ishiko, T. Relationships between muscle lactate accumulation and surface EMG activities during isokinetic contractions in man. Eur. J. Appl. Physiol. Occup. Physiol. 1987, 56, 18-23. [CrossRef]

19. Van Boerum, D.H.; Sangeorzan, B.J. Biomechanics and pathophysiology of flat foot. Foot Ankle Clin. 2003, 8, 419-430. [CrossRef]

20. Boozari, S.; Jamshidi, A.A.; Sanjari, M.A.; Jafari, H. Effect of functional fatigue on vertical ground-reaction force in individuals with flat feet. J. Sport Rehabil. 2013, 22, 177-183. [CrossRef]

21. Richie, D.H., Jr. Biomechanics and clinical analysis of the adult acquired flatfoot. Clin. Podiatr. Med. Surg. 2007, $24,617-644$. [CrossRef] [PubMed]

22. Beck, B.R.; Osternig, L.R. Medial tibial stress syndrome. The location of muscles in the leg in relation to symptoms. J. Bone Joint Surg. Am. 1994, 76, 1057-1061. [CrossRef] [PubMed]

23. Tweed, J.L.; Campbell, J.A.; Avil, S.J. Biomechanical risk factors in the development of medial tibial stress syndrome in distance runners. J. Am. Podiatr. Med. Assoc. 2008, 98, 436-444. [CrossRef] [PubMed] 
24. Yüksel, O.; Özgürbüz, C.; Ergün, M.; İşlegen, Ç.; Taskiran, E.; Denerel, N.; Ertat, A. Inversion/Eversion strength dysbalance in patients with medial tibial stress syndrome. J. Sports Sci. Med. 2011, 10, 737.

25. Solomonow, M.; Baten, C.; Smit, J.; Baratta, R.; Hermens, H.; D'Ambrosia, R.; Shoji, H. Electromyogram power spectra frequencies associated with motor unit recruitment strategies. J. Appl. Physiol. 1990, 68, 1177-1185. [CrossRef] [PubMed]

26. Kelly, L.A.; Cresswell, A.G.; Racinais, S.; Whiteley, R.; Lichtwark, G. Intrinsic foot muscles have the capacity to control deformation of the longitudinal arch. J. R. Soc. Interface 2014, 11, 20131188. [CrossRef] [PubMed]

27. Goo, Y.-M.; Heo, H.-J.; An, D.-H. EMG activity of the abductor hallucis muscle during foot arch exercises using different weight bearing postures. J. Phys. Ther. Sci. 2014, 26, 1635-1636. [CrossRef]

28. Lee, M.S.; Vanore, J.V.; Thomas, J.L.; Catanzariti, A.R.; Kogler, G.; Kravitz, S.R.; Miller, S.J.; Gassen, S.C. Diagnosis and treatment of adult flatfoot. J. Foot Ankle Surg. 2005, 44, 78-113. [CrossRef] 